\title{
Diagnostic Accuracy of Immunohistochemistry in Detecting MGMT Methylation Status in Patients with Glioma
}

\author{
Nita Sahara ${ }^{1}$, Rachmat Andi Hartanto ${ }^{2}$, Naomi Yoshuantari ${ }^{1}$, Kusumo Dananjoyo $^{3}$, \\ Irianiwati Widodo ${ }^{1}$, Rusdy Ghazali Malueka ${ }^{3}$, Ery Kus Dwianingsih ${ }^{1 *}$
}

\begin{abstract}
Background: The O6-methylguanine-DNA methyltransferase (MGMT) gene prevents mismatch in DNA replication and transcription by repairing mutagenic DNA lesions. MGMT is a predictor biomarker of chemotherapy in high-grade and low-grade gliomas based on high-risk clinical conditions. It also can be used for therapeutic decisions to predict hypermutation in recurrence in newly diagnosed low-grade gliomas. The gold standard examination for the methylation is Polymerase Chain Reaction (PCR). However, this technique is not widely available in Indonesia for daily practice. Thus, an uncomplicated and simpler method such as immunohistochemistry (IHC) is needed as an alternative examination. This study aimed to predict the diagnostic accuracy of immunohistochemistry (IHC) in detecting the methylation status of O6-methylguanine-DNA methyltransferase (MGMT) in glioma. Methods: This research was a cross-sectional study using formalin-fixed paraffin embedded (FFPE) tissue samples of glioma patients, dating between October 2017 until March 2021. Diagnosis of glioma was established based on clinical, radiological, and histopathological findings. MGMT methylation status was investigated using the IHC and PCR techniques. Diagnostic value of IHC was analyzed, with PCR as a gold standard method. Optimum threshold to determine positivity of IHC was determined by the Area Under the Curve (AUC) on Receiver Operating Characteristics (ROC) curve and Youden index. Results: Among 75 samples examined, 29 (38.7\%) patients were methylated. IHC detected $M G M T$ methylation with sensitivity of $86.2 \%$, specificity of $63.0 \%$, positive predictive value of $59.5 \%$, negative predictive value of $87.9 \%$ and accuracy of $72.0 \%$. The AUC was 0.746 , indicating moderate diagnostic value. Optimum positivity threshold of the IHC examination based on Youden Index was 10\%. Conclusion: IHC examination can be used to detect MGMT methylation status of glioma patients in limited resources setting, where PCR technique is not available.
\end{abstract}

Keywords: Glioma- methylated MGMT- IHC- PCR- diagnostic accuracy

Asian Pac J Cancer Prev, 22 (12), 3803-3808

\section{Introduction}

Glioma is the most often found primary intracranial tumor with the highest incidences occurring in Europe (5.5 per 100,000 population), North America (5.3 per 100,000 population), Australia (5.3 per 100,000 population), North Africa (5 per 100,000 population) and West Asia (5.2 per 100,000 population). The incidence of this disease has remained relatively unchanged in the past decade (Ostrom et al., 2017). Life expectancy of patients with glioma is still very low and the death rate is still high with a median survival rate of glioblastoma only ranging from 11-12 months (Bohn et al., 2018). Histomorphology, genetic, and epigenetic profiles of tumors are thought to play a role in determining the success of therapy and the high and low life expectancy of gliomas.

According to the World Health Organization (WHO), glioma is classified into grades I, II, III, and IV. Current classification of the tumors includes the mutation status of IDH gene considering whether it is mutant, wild type, or no otherwise specified (NOS) for the tumor that is not examined for the mutation status yet. The mutation status of IDH is an important prognostic factor in glioma (WHO, 2016).

Several studies have shown that O6-methylguanineDNA methyltransferase (MGMT) promoter methylation can predict the benefits of alkylation chemotherapy and can be used as a predictive biomarker for targeted therapy (Reifenberger et al., 2017). The function of MGMT is very important for the stability of the genome. MGMT is a tumor suppressor gene that is involved in mutagenic DNA repairment and prevents mismatches or errors during DNA replication and transcription (Pfeifer, 2018). The methylation of the $M G M T$ promoter region has been stated

${ }^{1}$ Department of Anatomical Pathology, Faculty of Medicine, Public Health and Nursing, Universitas Gadjah Mada, Indonesia. ${ }^{2}$ Division of Neurosurgery, Department of Surgery, Faculty of Medicine, Public Health and Nursing, Universitas Gadjah Mada, Indonesia. ${ }^{3}$ Department of Neurology, Faculty of Medicine, Public Health, and Nursing, Universitas Gadjah Mada (FK-KMK UGM), Dr. Sardjito General Hospital, Yogyakarta, Indonesia.*For Correspondence: ery_malueka@ugm.ac.id 
as one of strongest predictors of the temozolomide (TMZ) treatment by promoting prevention of gene translation, thereby halting DNA repair in cancer cells (Sze et al., 2013).

Currently, the MGMT examination has been applied as a biomarker predictor of chemotherapy in high-grade and low-grade gliomas based on high-risk clinical conditions (Bell et al., 2018). MGMT promoter methylation can also be used as a potential biomarker for therapeutic decisions, where it predicts hypermutation in recurrence in newly diagnosed low-grade gliomas (Mathur et al., 2013).

Studies have shown that the polymerase chain reaction (PCR) technique has high sensitivity, specificity, and accuracy in detecting MGMT methylation (Wang et al., 2017). However, this technique is challenging to be applied in Indonesia since it is not yet widely used in daily practice and is expensive. The $M G M T$ silencing in glioma is associated with hypermethylation of the $M G M T$ in the promoter region and can be detected at the protein level using immunohistochemical (IHC) techniques which is cheaper and simpler than PCR (Quillien et al., 2012). Thus, the IHC technique can be proposed as an alternative diagnostic method to give proper management for glioma's cases in Indonesia. This study aimed to predict the diagnostic accuracy of IHC technique in detecting $M G M T$ methylation status in glioma.

\section{Materials and Methods}

\section{Samples and data collection}

Seventy-five subjects were enrolled between October 2017 until March 2021 in Dr. Sardjito and Klaten General Hospitals, Yogyakarta, Indonesia. Glioma patients at all grades who agreed to participate in the study and underwent surgical tumor removal were recruited. Tumor samples were collected for histopathological examination to establish the diagnosis. The leftover Formalin-Fixed Paraffin Embedded (FFPE) samples were sliced for immunostaining.

In addition, fresh tissue samples of the tumor that came from the same part of tumor submitted to Pathology Department were collected in the Biobank Facility of the Faculty of Medicine, Public Health and Nursing, Universitas Gadjah Mada for DNA extraction. Clinical and demographic data were collected through medical records. The study was approved by the Medical and Health Research Ethics Committee of the Faculty of Medicine, Public Health and Nursing Universitas Gadjah Mada, Yogyakarta, Indonesia with approval number: (KE/ FK/0115/EC/2020).

\section{Immunostaining}

The samples were cut into $4 \mu \mathrm{m}$-thick sections, which were transferred to an incubator at $37^{\circ} \mathrm{C}$ for one night, or incubated by placing on a hotplate at $60^{\circ} \mathrm{C}$ for 10 minutes then deparaffinized, and rehydrated. Mouse monoclonal antibody MGMT (MT3.1) ab39253 was used in this study. Antigen retrieval procedure was performed using a decloaking chamber. Primary antibody was diluted to 1:50 in phosphate buffer saline, and stained with Hematoxylin as a counter staining. Tonsil tissue was used as positive control.

\section{Interpretation of MGMT immunostaining}

The results of immunohistochemical staining were assessed by two (Bohn et al., 2018) observers using a conventional light microscope. Brown nuclear staining in any intensity of tumor cell was considered as a positive staining. Qualitative calculation of tumor nuclear staining was determined by eye ball, with a cut-off point of $10 \%$ as previously described by Wang et al. (2017). Nuclear staining of $\geq 10 \%$ of tumor cells was interpreted as immunopositive, while the opposite result was interpreted as immunonegative (Figure 1). Diagnostic value was analyzed by Area Under the Curve (AUC) on the Receiver Operating Characteristics (ROC) curve. Each specimen value of nuclear IHC staining percentage was also analyzed by Youden index to determine the optimum cutoff of the IHC positivity threshold.

\section{Polymerase Chain Reaction Examination}

The DNA was extracted from available FFPE or fresh tissue samples. The FFPE sample was deparaffinized and the DNA was isolated using the QIAamp DNA FFPE Tissue Kit (QIAGEN, Cat. \#56404, Hilden, Germany). The DNA from fresh tissue samples was extracted using the Quick DNA FFPE MiniPrep Kit (Zymo Research, USA). The NanoVue Plus spectrophotometer (GE Healthcare Life Sciences, Pittsburgh, PA, USA) was used to estimate the recovered DNA (Malueka et al., 2020). The EZ DNA Methylation-Gold Kit (Zymo Research Cat o. D5005 Lot No. ZRC 200814) was used to convert unmethylated cytosine to uracil (bisulfite conversions) with bisulfite treatment of up to $300 \mathrm{ng}$ of DNA. The converted DNA was used (125 ng) for quantitative real-time PCR (qRT-PCR) analysis of $M G M T$ promoter methylation (Rivera et al., 2010). The amplification of methylation-specific qRT-PCR represents the methylation status of the $M G M T$ promoter region. The interpretation of methylated status was marked if the green curve was under red curve, while unmethylated status was interpreted if the blue curve was under red curve (Figure 2). The red curve serves as an internal control of COL2A1 (Malueka et al., 2020).

\section{Statistical analysis}

Sensitivity, specificity, positive predictive value, negative predictive value and accuracy of IHC were analyzed using a 2x2 table, with PCR technique as a gold standard. Association between IHC and PCR examination results on $M G M T$ methylation was analyzed using chisquare tests.

\section{Results}

\section{Patient characteristics}

Among 75 patients, $44(58.7 \%)$ were male and 31 samples $(41.3 \%)$ were female. Mean of patients' age was 44.8 years, the youngest was 2 years, whereas the oldest was 73 years. Most of the patients $(56.0 \%)$ were in the age of $<50$ years. 
Table 1. Characteristics of Glioma Patients Based on Methylation Status by PCR Method

\begin{tabular}{|c|c|c|}
\hline \multirow[t]{3}{*}{ Variable } & \multicolumn{2}{|c|}{ MGMT Methylated Status by PCR } \\
\hline & Methylated & Unmethylated \\
\hline & \multicolumn{2}{|c|}{$\mathrm{n}(\%)$} \\
\hline \multicolumn{3}{|l|}{ Gender } \\
\hline Male & $19(61.5)$ & $25(54.3)$ \\
\hline Female & $10(34.5)$ & $21(45.7)$ \\
\hline \multicolumn{3}{|l|}{ Age (year) } \\
\hline$\geq 50$ & $8(27.6)$ & $25(54.3)$ \\
\hline$<50$ & $21(72.4)$ & $21(45.7)$ \\
\hline \multicolumn{3}{|l|}{ Grading Glioma } \\
\hline I & $0(0.0)$ & $1(2.2)$ \\
\hline II & $5(17.2)$ & $10(21.7)$ \\
\hline III & $7(24.1)$ & $11(23.9)$ \\
\hline IV & $17(58.6)$ & $24(52.2)$ \\
\hline \multicolumn{3}{|l|}{ Grading Glioma } \\
\hline High Grade & $24(82,8)$ & $33(71.7)$ \\
\hline Low grade & $5(17.2)$ & $13(28.3)$ \\
\hline$\%$ of total & $29(38.7)$ & $46(61.3)$ \\
\hline
\end{tabular}

Tumor grade

Most of glioma samples in this study were classified into high grade. As many as As many as 41 samples $(54.7 \%)$ were grade IV, 18 samples $(24.0 \%)$ grade III, 15 samples $(20.0 \%)$ grade II, and 1 sample $(1.3 \%)$ grade I. Glioblastoma, NOS was the most common diagnosis (41 samples, 54,6\%)

\section{MGMT methylation status using IHC}

Interobserver agreement in IHC examination was analyzed using Cohen's kappa value measurement and showed the value of 0.842 . Among 75 samples, 71 of them $(94.6 \%)$ were interpreted consistently between 2 observers. Negative immunostaining (methylated MGMT promoter) was found in 42 samples $(56.0 \%)$, whereas 33 samples $(44.0 \%)$ showed positive immunostaining (unmethylated $M G M T$ promoter). Immuno-expression was regarded as methylated when the IHC showed an immuno-negative result with unstained tumor cells nuclei, or stained in $<10 \%$ of the tumor cell population. Meanwhile, unmethylated result was defined if the result showed the opposite feature (Figure 1).

\section{MGMT methylation status using PCR}

Methylated MGMT promoter sequence was considered to represent the methylation status of the $M G M T$ promoter on the tumor and was marked by a green curve, while the unmethylated $M G M T$ promoter was marked with a blue curve (Figure 2). Positive MGMT methylation status was found in 29 samples (38.7\%), whereas negative status was found in 46 samples (61.3\%) (Table 1.).

Methylation status was more common in men (19 samples, $61.5 \%$ ) than women (10 samples, $34.5 \%$ ). The group of $<50$ years old had more methylation than the group of $>50$ years old ( 21 samples, $72.4 \%$ ). In line with these results, methylation status was also more common in high grade gliomas (24 samples, $82.8 \%$ ) compared to low grade ( 5 samples, $17.2 \%$ ). Glioma grade IV showed the highest number of methylation status (17 samples, 58.6\%) (Table 1).

\section{Diagnostic value analysis}

Among 42 samples with positive methylation status using the IHC method, 25 samples (59.5\%) were concordant with the PCR results, whereas 17 samples $(40.5 \%)$ were not. Among 33 samples with negative
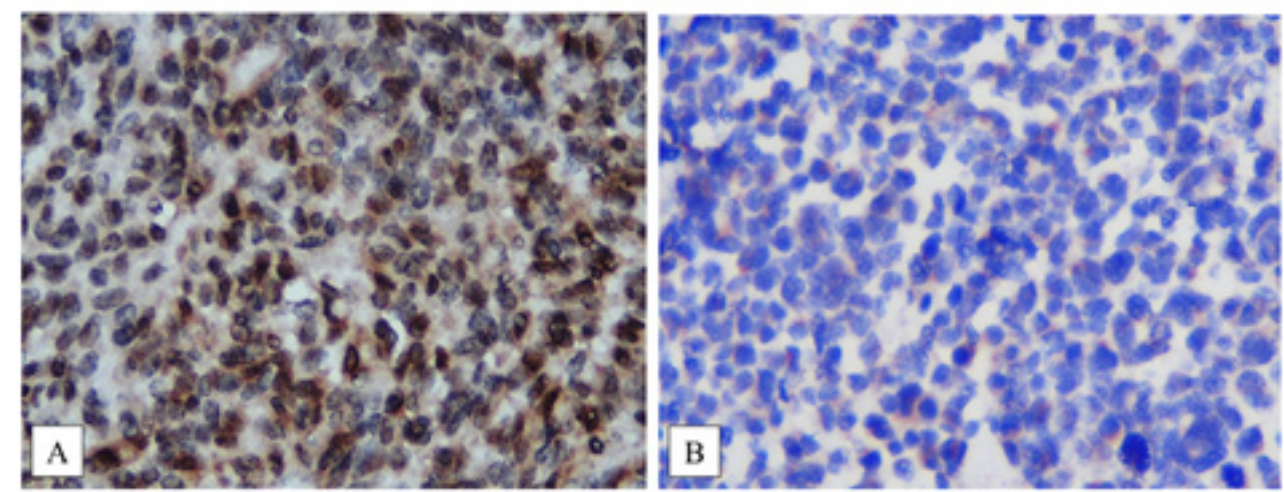

Figure 1. Immunohistochemical Results of $M G M T$ Methylation (magnification 400x). (A). Unmethylated status was determined by a positive nuclear staining of the tumor using anti-MGMT antibody (MT3.1) ab39253 which was assessed quantitatively using eye balling technique with a cut-off of $\geq 10 \%$. (B). Methylated status was determined by a negative nuclear staining with cut-off $<10 \%$.

Table 2. IHC Results Compared to PCR Method

\begin{tabular}{llccc}
\hline & & \multicolumn{2}{c}{ PCR } & N (\% within total) \\
\multicolumn{1}{l}{ Methylated } & Unmethylated & \\
\hline IHC & Methylated (negative expression) & $25(59.5)$ & $17(40.5)$ & $42(56.0)$ \\
& Unmethylated (positive expression ) & $4(12.1)$ & $29(87.9)$ & $33(44.0)$ \\
\hline
\end{tabular}




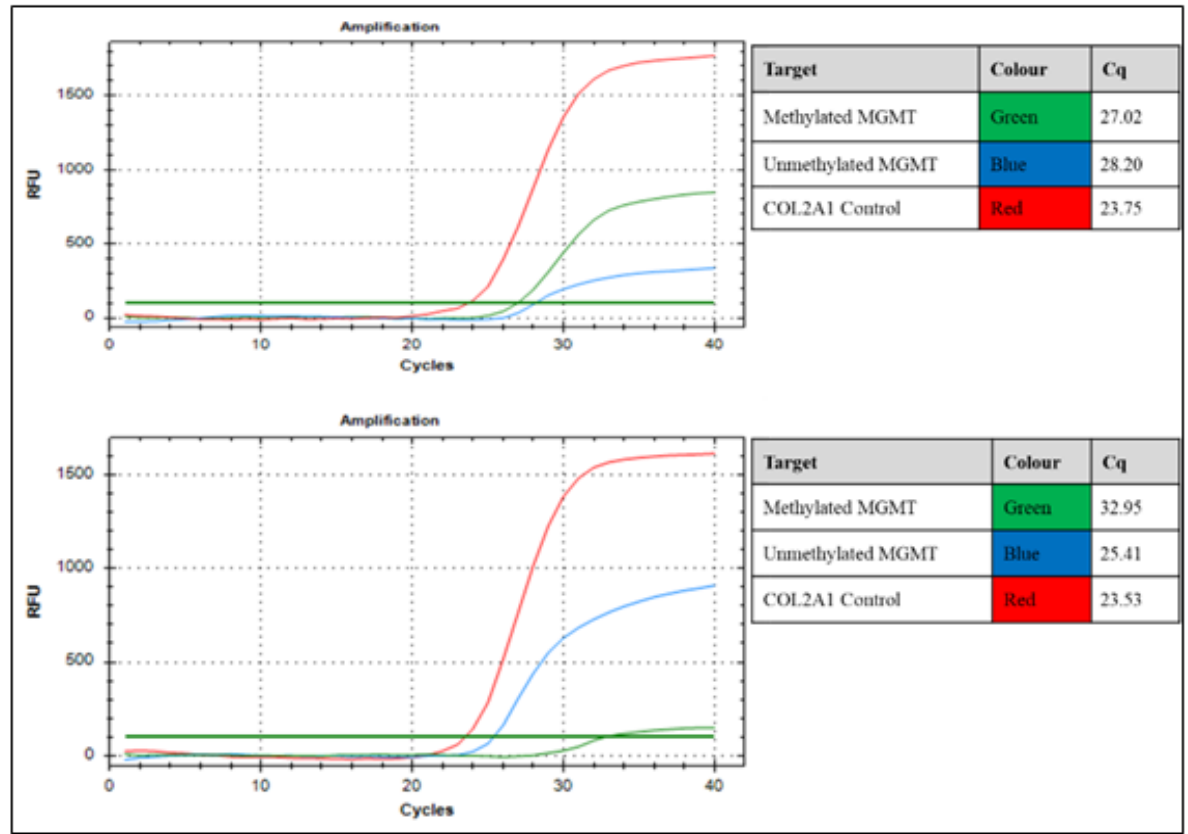

Figure 2. Methylation-specific PCR Curve Shows the Amplification of MGMT with COL2A1 as internal control (red), methylated MGMT gene promoter (green) and unmethylated MGMT gene promoter (blue); (A) Methylated status is marked with green curve under red curve; (B) Unmethylated is interpreted if blue curve under red curve.

Table 3. Cut-off Value of Nuclear Immunostaining Percentage in MGMT Methylation Status Based on Youden Index $(\mathrm{J})$

\begin{tabular}{lccc}
\hline Cut-off $(\%)$ & Sensitivity & Specificity & J \\
\hline 1 & 0.48 & 0.67 & 0.15 \\
5 & 0.72 & 0.63 & 0.35 \\
10 & 0.86 & 0.63 & 0.49 \\
15 & 0.86 & 0.47 & 0.33 \\
20 & 0.86 & 0.47 & 0.33 \\
25 & 0.86 & 0.41 & 0.27 \\
30 & 0.86 & 0.41 & 0.27 \\
40 & 0.89 & 0.32 & 0.21 \\
50 & 0.93 & 0.28 & 0.21 \\
\hline
\end{tabular}

methylation status using the IHC method, 29 samples $(87.9 \%)$ were concordant with PCR results, whereas only 4 samples (12.1\%) were not (Table 2).

The results of the statistical test showed that the AUC value from the ROC curve was 0.746 which was statistically significant in the form of the IHC method having moderate diagnostic value strength $(>70 \%-80 \%)$ (Figure 3).

The optimum threshold to determine positivity of the nuclear IHC staining percentage was determined by Youden Index. The highest index was found in 10\% nuclear staining. Thus, we propose that specimens with nuclear IHC staining in $\leq 10 \%$ cells were considered $M G M T$ methylated and specimens with nuclear IHC staining in

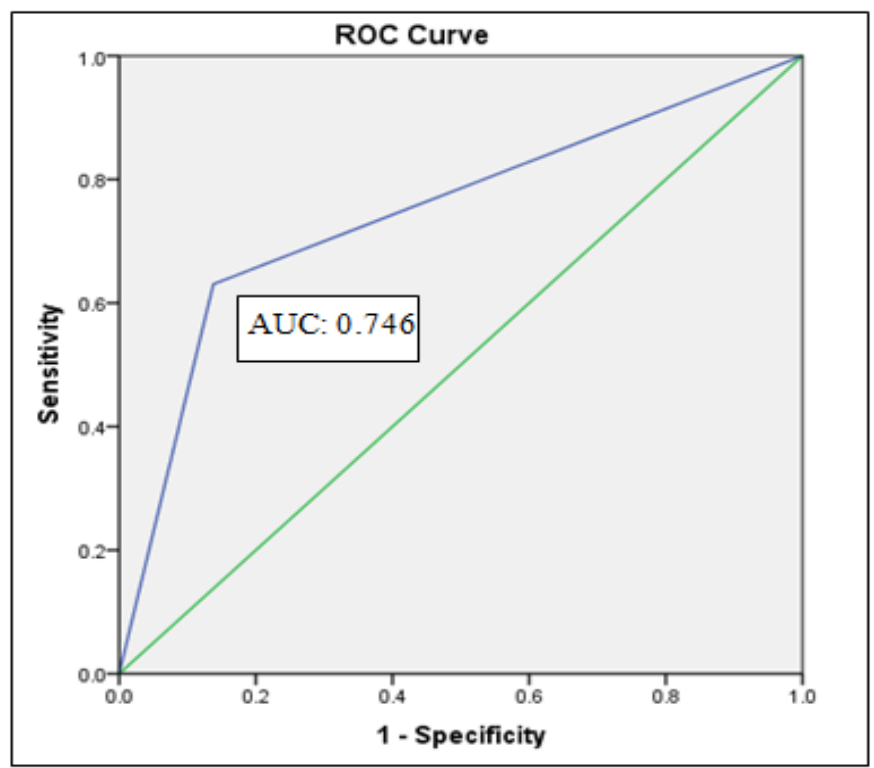

Figure 3. AUC Value was 0.746, Indicating Moderate Diagnostic Value on the ROC Curve 
Table 4. Diagnostic Value of IHC Compared to PCR in Detecting MGMT Methylation Status

\begin{tabular}{lccccc}
\hline & Sensitivity (\%) & Specificity (\%) & PPV (\%) & NPV (\%) & Accuracy (\%) \\
\hline IHC & 86.2 & 63.0 & 59.5 & 87.9 & 72.0 \\
\hline
\end{tabular}

$>10 \%$ cells were considered $M G M T$ unmethylated (Table 3). Diagnostic value calculations showed that IHC detects MGMT methylation with sensitivity $86.2 \%$, specificity $63.0 \%$, positive predictive value $59.5 \%$, negative predictive value $87.9 \%$ and accuracy $72.0 \%$ (Table 4 ).

\section{Discussion}

MGMT promoter methylation was identified in $38.7 \%$ of the samples, which was less than the unmethylated ones. This result is concordant with previous study that reported $37.8 \%$ of the glioma harbored $M G M T$ promoter methylation. The slight difference in proportion can be attributed to tumor heterogeneity of the samples (Mellai et al., 2012). In this study, the accuracy of the IHC technique was $72.0 \%$ which is similar with previous studies that showed accuracy of 78\% (Kristensen et al., 2016) and is even higher than a study by Hsu (2017) which showed only $49.4 \%$ accuracy.

MGMT methylation was mostly found in grade IV of glioma which is similar with previous research that showed high methylation status in glioblastoma (WHO grade IV) (Wang et al., 2017). However, these findings are contrary with a study by Li et al., (2017) that showed no association between methylation status of $M G M T$ with tumor grade, age, and sex. Li et al., (2017) also reported that $M G M T$ expression was higher in low grade glioma and lower in high grade glioma. There is still no solid consensus concerning the correlation between MGMT methylation status and glioma grade yet.

This study showed that AUC was 0.746 , representing a moderate diagnostic value of IHC in determining MGMT methylation status. IHC was considered as a potentially powerful diagnostic tool to assess $M G M T$ expression, since it has significant negative association with patients' survival in diffuse astrocytoma and glioblastoma (Capper et al., 2008). One study conducted by Pandith et al. reported a significant association between IHC and PCR results in detecting $M G M T$ methylation status in glioma. $M G M T$ gene promoter methylation led to the inactivation of its subsequent protein. Thus, methylation of $M G M T$ can be detected by its loss of protein expression (Pandith et al., 2018). However, Rodriguez (2008) reported an insignificant correlation between MGMT immunohistochemical expression and promoter methylation in human glioblastoma. This difference in findings can be attributed to confounding factors in the IHC technique, such as interobserver agreement, immunostaining protocol, different manufacturers of antibody and antibody clonality. Moreover, non-tumor cells, such as endothelial cells, lymphocytes, macrophages, and microglial cells, can be stained positively by IHC. This outcome may also lead to false positive interpretations. Tumor heterogeneity is also a factor that can contribute to different results across studies (Lipp et al., 2019).

Sensitivity and negative predictive value of IHC to detect $M G M T$ methylation in this study are $86.2 \%$ and $87.9 \%$, respectively. These results were concordant with a study in India that reported sensitivity and negative predictive value of IHC were $81.6 \%$ and $86.1 \%$, respectively (Pandith et al., 2018). In this study, specificity and positive predictive value were $63.0 \%$, and $59.5 \%$, respectively. On the other hand, a previous study reported higher specificity and positive predictive values of IHC as $77.3 \%$ and $70.8 \%$, respectively (Pandith et al., 2018). These differences can be caused by different manufacturers of antibodies, immunostaining protocols, various cut-off points, small tumor sampling, wide necrotic area and tumor heterogeneity (Brell et al., 2011).

In this study, a high value of sensitivity, with relatively low positive predictive value indicated that the methylated MGMT status using IHC still needs to be confirmed with PCR examination. Some studies recommended DNA sequencing to detect $M G M T$ methylation. However, the number of medical centers and hospitals which have the facility of sequencing technology is still very limited. Moreover, laboratory cost of using a sophisticated technology is still not affordable for most patients. A simpler and cost-effective method, such as IHC, is necessary to be applied in daily practice, especially in health centers that do not have PCR or sequencing facilities.

In conclusion, this study showed a moderate diagnostic value of IHC compared with the PCR method in detecting MGMT methylation status in glioma patients. IHC method is sufficient as a modality to detect $M G M T$ methylation status in limited resources setting where the PCR technique is not available.

\section{Author Contribution Statement}

The authors confirm contribution to the paper as follows: study conception and design: Ery Kus Dwianingsih, Rusdy Ghazali Malueka; data collection: Rachmat Andi Hartanto; Kusumo Dananjoyo; analysis and interpretation of results: Nita Sahara, Naomi Yoshuantari, Irianiwati Widodo; draft manuscript preparation: Nita Sahara, Rusdy Ghazali Malueka, Ery Kus Dwianingsih. All authors reviewed the results and approved the final version of the manuscript.

\section{Acknowledgements}

We are thankful to Sri Fatmawati and Agustina for their great contribution in assisting laboratory work for this study. We are also grateful for the language editing service at Klinik Bahasa FK-KMK UGM for the English proofreading of this manuscript.

\section{Funding Statement}

This study was supported by a grant from the Ministry of Research, Technology, and Higher Education 
2017-2021.

\section{References}

Bell EH, Zhang P, Fisher BJ, et al (2018). Association of MGMT promoter methylation status with survival outcomes in patients with high-risk glioma treated with radiotherapy and temozolomide: an analysis from the NRG Oncology/RTOG 0424 Trial. JAMA Oncol, 4, 1405-9.

Bohn A, Braley A, Vega PR, et al (2018). The association between race and survival in glioblastoma patients in the US: A retrospective cohort study. PLoS ONE, 13, 1-10.

Brell M, Ibáñez J, Tortosa A (2011). O6-MethylguanineDNA methyltransferase protein expression by immunohistochemistry in brain and non-brain systemic tumours: Systematic review and meta-analysis of correlation with methylation-specific polymerase chain reaction. BMC Cancer, 11, 5-7.

Capper D, Mittelbronn M, Meyermann R, et al (2008). Pitfalls in the assessment of MGMT expression and in its correlation with survival in diffuse astrocytomas: Proposal of a feasible immunohistochemical approach. Acta Neuropathol, 115, 249-59.

Kristensen LS, Michaelsen SR, Dyrbye H, et al (2016). Assessment of quantitative and allelic MGMT methylation patterns as a prognostic marker in glioblastoma. J Neuropathol Exp Neurol, 75, 246-55.

Li Q, Guo J, Wang W, et al (2017). Relationship between MGMT gene expression and treatment effectiveness and prognosis in glioma. Oncol Letters, 14, 229-233.

Lipp ES, Healy P, Austin A, et al (2019). MGMT: Immunohistochemical detection in high-grade astrocytomas. J Neuropathol Exp Neurol, 78, 57-64.

Malueka RG, Dwuianingsih ER, Bayuangga HF, et al (2020). Clinicopathological features and prognosis of Indonesian patients with gliomas with IDH mutation: Insights into its significance in a Southeast Asian population. Asian Pac J Cancer Prev, 21, 2287-95.

Mathur R, Zhang Y, Grimmer MR, et al (2020). MGMT promoter methylation level in newly diagnosed low-grade glioma is a predictor of hypermutation at recurrence. J Neuro-Oncol, 22, 1580-90.

Mellai M, Monzeglio O, Piazzi A, et al (2012). MGMT promoter hypermethylation and its associations with genetic alterations in a series of 350 brain tumors. J Neuro-Oncol, 107, 617-31.

Ostrom QT, Gittleman H, Stetson L, et al (2017). Epidemiology of Intracranial Gliomas. Prog Neurol Surg, 30, 1-11.

Pandith AA, Qasim I, Zahoor W, et al (2018). Concordant association validates MGMT methylation and protein expression as favorable prognostic factors in glioma patients on alkylating chemotherapy (Temozolomide). Scientific Reports, 8, 1-11.

Pfeifer GP (2018). Defining driver DNA methylation changes in human cancer. Int J Mol Sci, 19, 1-13.

Quillien V, Lavenu A, Karayan-Tapon L, et al (2012). Comparative assessment of 5 methods (methylation-specific polymerase chain reaction, methylight, pyrosequencing, methylation-sensitive high-resolution melting, and immunohistochemistry) to analyze O6-methylguanineDNA- methyltranferase in a series of 100 . Cncr, 118, 4201-11.

Reifenberger G, Wirsching HG, Knobbe-Thomsen CB, Weller $\mathrm{M}$ (2017). Advances in the molecular genetics of gliomasimplications for classification and therapy. Nature Rev Cli Oncol, 14, 434-52.

Rivera AL, Pelloskit CE, Gilbert MR, et al (2010). MGMT promoter methylation is predictive of response to radiotherapy and prognostic in the absence of adjuvant alkylating chemotherapy for glioblastoma. Neuro-Oncol, $12,116-21$.

Sze CI, Su, WP, Chiang, MF, et al (2013). Assessing current therapeutic approaches to decode potential resistance mechanisms in glioblastomas. Frontiers Oncol, 3, 1-7.

Wang L, Li Z, Liu C, et al (2017). Comparative assessment of three methods to analyze MGMT methylation status in a series of 350 gliomas and gangliogliomas. J PRP, 213, 1489-93.

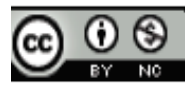

This work is licensed under a Creative Commons AttributionNon Commercial 4.0 International License. 